\title{
Conocimiento especializado de un profesor de Educación Infantil al enseñar cuerpos geométricos
}

\author{
Specialised knowledge of an early childhood education teacher when teaching geometric \\ solids
}

\author{
Ana María Escudero Domínguez ${ }^{1}$ \\ María de la Cinta Muñoz-Catalán ${ }^{2}$ \\ José Carrillo Yáñez $z^{3}$
}

\begin{abstract}
Resumen
Este trabajo forma parte de una investigación más amplia que tiene como objetivo comprender el conocimiento de un profesor de Educación Infantil al enseñar cuerpos geométricos. Para dar sustento teórico a la investigación aquí mostrada usamos el modelo Mathematics Teacher's Specialised Knowledge (MTSK), con el cual obtenemos indicadores de conocimiento especializado del profesor de Educación Infantil. Se trata de un estudio de caso instrumental cualitativo enfocado desde un paradigma interpretativo. Los resultados ponen en evidencia la complejidad del conocimiento del profesor en la etapa de Educación Infantil. Mostramos evidencias e indicios del conocimiento especializado de nuestro informante, poniendo de manifiesto la profundidad de los conocimientos matemáticos y del conocimiento didáctico del contenido que posee, así como las relaciones entre conocimientos de distinta índole.
\end{abstract}

Palabras clave: Conocimiento del profesor; Cuerpos geométricos; Educación Infantil; Estudio de caso

\begin{abstract}
This work is part of a broader investigation that aims to understand the knowledge of an Early Childhood Education teacher when teaching geometric solids. To give theoretical support to the research shown here, we use the Mathematics Teacher's Specialised Knowledge model (MTSK), with which we obtain indicators of specialised knowledge from the Early Childhood Education teacher. It is an instrumental case study, qualitative in nature, focused from an interpretive paradigm. The results show the complexity of the teacher's knowledge in the Early Childhood Education. We show evidence and indications of the specialised knowledge of our informant, highlighting the depth of the mathematical knowledge and pedagogical content knowledge he possesses, as well as the relationships between knowledge of different kinds.
\end{abstract}

Keywords: Knowledge of the teacher; Geometric solids; Early Childhood Education; Case study

Submetido em: 30/10/2020 - Aceito em: 23/04/21 - Publicado em: 27/05/2021

${ }^{1}$ Doctora en Didáctica de la Matemática. Profesora-Investigadora de la Universidad de Sevilla. España. Email: aescudero1@us.es. ORCID: https://orcid.org/0000-0002-1620-3852

2 Doctora en Didáctica de la Matemática. Profesora Contratada Doctora de-Investigadora de la Universidad de Sevilla. España. Email: mcmunozcatalan@us.es. ORCID: https://orcid.org/0000-0003-2329-7612

3 Doctor en Didáctica de la Matemática. Catedrático de la Universidad de Huelva. España. Email: carrillo@uhu.es. ORCID: https://orcid.org/0000-0001-7906-416X 


\section{Introducción y antecedentes}

En los últimos años se ha producido un auge en la investigación relacionada con la etapa de Educación Infantil, asumiendo que los primeros años de escolarización constituyen los cimientos de los aprendizajes matemáticos futuros (McCray \& Chen, 2012). Existe aún poca investigación con respecto al profesor de esta etapa educativa (Parks \& Wager, 2015), ya que suele pensarse que las matemáticas en Educación Infantil las puede enseñar cualquiera (Castro \& Castro, 2016). Aunque asociaciones profesionales como la National Association for the Education of Young Children y el National Council of Teachers of Mathematics (NAEYC \& NCTM, 2013) establecen la necesidad de que los profesores de la etapa de Infantil posean una formación matemática sólida a través de la cual puedan mostrar las bases para el desarrollo de la formación matemática temprana en estos discentes (Muñoz-Catalán, Liñán \& Ribeiro, 2017). En esa línea, Perry y Dockett (2002) afirman que estos profesores deben conocer las ideas matemáticas profundas en las que se basan las tareas que se realizan en esta etapa, aunque estas parezcan simples (Pitta-Pantazi \& Christou, 2011). El profesor de Educación Infantil no es especialista en ningún área, por lo que no podemos hablar del profesor de matemáticas en Educación Infantil. Aunque el currículo español no establece como área las matemáticas, este profesional necesita disponer de un conocimiento sólido para identificar las matemáticas fundamentales que sirven de cimientos para aprendizajes matemáticos en etapas posteriores.

Una de las principales características de la enseñanza de Educación Infantil es que los contenidos escolares se plantean de forma lúdica (Wernberg, Larsson \& Riesbeck, 2010), ya que se trabaja con el juego guiado con el objetivo de alimentar la indagación de los niños, su compromiso e interés por las matemáticas. Otra característica de la práctica profesional de este docente es que debe tener presente que se trata de una etapa generadora de lenguaje (Escudero-Domínguez, Escudero-Ávila, Aguilar-González \& Vasco-Mora, 2019). Por lo cual entendemos que los profesores han de ofrecer a los niños oportunidades para pensar y expresarse por sí mismos, con su propio lenguaje, y una vez tengan interiorizado el concepto, irles ofreciendo el vocablo formal. Por otra parte, hay que tener en cuenta que el tratamiento de conceptos que se va trabajando en la etapa se realiza de forma helicoidal.

El aprendizaje de la geometría es imprescindible en estas primeras edades debido a la ayuda que proporciona para que los niños logren comprender el espacio en el que se mueven (Clements \& Sarama, 2011). La adquisición de conocimientos geométricos contribuye al desarrollo de la competencia matemática y del razonamiento lógico, lo que a su vez potencia el desarrollo de habilidades para visualizar, pensar críticamente, intuir, resolver problemas y conjeturar (Gamboa \& Ballestero, 2010). Sin embargo, a pesar del papel de la geometría en el aprendizaje de los alumnos, durante años no se le ha prestado la atención que merecía (Clements \& Sarama, 2011).

Revisando las actas de los principales congresos en el área del periodo 2010-2018, se observa que la investigación sobre el conocimiento profesional del profesor que enseña matemáticas en Educación Infantil todavía es incipiente. En una de ellas, se toma como modelo teórico de 
análisis el Mathematical Knowledge for Teaching [en adelante MKT] (Ball, Thames, \& Phelps, 2008) pero se concluye que el marco teórico debe ajustarse a la etapa de Educación Infantil para ser utilizado en ese entorno (Mosvold, Bjuland, Fauskanger \& Jacobsen, 2011). En los últimos años, encontramos otra investigación acerca del conocimiento revelado por una profesora de Educación Infantil sobre la enseñanza de la Geometría en un aula de 5 años (Hundeland, Erfjord \& Carlsen, 2017). Este estudio utiliza la lente del Knowledge Quartet [en adelante KQ] (Rowland, Huckstep \& Thwaite, 2005) como herramienta para observar el conocimiento que entra en juego en el aula y concluye que el KQ se desarrolla de forma diferente en el contexto de Educación Infantil, pero que es útil para caracterizar el conocimiento del profesor de esta etapa. Desde el año 2017 encontramos distintos trabajos (Policastro, Almeida \& Ribeiro, 2017; Muñoz-Catalán, et al., 2019; Escudero-Domínguez, Muñoz-Catalán \& Carrillo, en prensa, entre otros) que intentan avanzar en la comprensión del conocimiento especializado del profesor de Educación Infantil en la enseñanza de las matemáticas. En estas investigaciones queda patente cómo el modelo Mathematics Teacher's Specialised Knowledge [en adelante MTSK] (Carrillo, et al., 2018) es una herramienta útil para reflexionar sobre el conocimiento especializado del profesor de Educación Infantil. Los distintos análisis muestran aspectos como la densidad y cohesión que definen el conocimiento especializado del profesor de Educación Infantil (Muñoz-Catalán, et al., 2017). Estos trabajos muestran como los elementos de MK son importantes en este profesional y cómo estos sostienen al desarrollo de las dimensiones del PCK (Policastro, et al., 2017; EscuderoDomínguez, et al., en prensa, entre otros). Por ejemplo, el conocimiento de distintos sistemas de representación y la complementariedad entre ellos (KoT) es clave para tomar decisiones sobre los recursos didácticos (KMT). Y el conocimiento de la comparación como práctica matemática que promueve conocimiento matemático en la etapa (KPM) es usada como estrategia de enseñanza (KMT) (Muñoz-Catalán, et al., 2019). Estas relaciones nos vislumbran la existencia de elementos de conocimientos de distinta índole, así como la diversidad de relaciones entre los distintos subdominios (Zakaryan \& Ribeiro, 2016).

Teniendo como horizonte profundizar en el desarrollo del MTSK en Educación infantil, en este artículo pretendemos comprender el conocimiento especializado movilizado por un profesor de Educación Infantil en relación con la enseñanza de los cuerpos geométricos.

\section{Fundamentación Teórica}

El conocimiento del profesor se ha estudiado durante décadas, siendo Shulman (1986) quien planteó la necesidad de considerar la propia disciplina para caracterizar el conocimiento profesional e introdujo una distinción entre conocimiento del contenido y conocimiento didáctico del contenido. Sobre esta base, Ball, Thames, \& Phelps (2008) desarrollaron el modelo Mathematical Knowledge for Teaching el cual plantea dificultades para la etapa de Infantil porque su aplicación está fuertemente condicionada por el enfoque pedagógico del contexto cultural en el que fue creado. Así, en el contexto americano en el que la etapa de Infantil está orientada a la enseñanza como preparación para Primaria, las 
DOI: 10.20396/zet.v29i00.8661819

tareas que definen el trabajo de enseñanza del profesor de matemáticas (como presentar ideas matemáticas o encontrar un ejemplo para ilustrar una idea concreta) permiten la aplicación del modelo directamente. En otros contextos, como el noruego, por ejemplo, con un enfoque pedagógico más social, con otro papel atribuido al profesor, esta aplicación es más problemática como detectaron (Mosvold, et al., 2011). Lo anterior, unido a nuestra consideración de que la especificidad del conocimiento del profesor en relación con la enseñanza de las matemáticas no se limita a un solo subdominio, nos llevó a utilizar el Mathematics Teacher's Specialised Knowledge [MTSK] (Carrillo, et al., 2018). Este modelo se caracteriza por considerar la especialización del conocimiento del profesor de matemáticas en cada uno de los subdominios que lo componen (Scheiner, Montes, Godino, Carrillo \& Pino-Fan, 2019). El modelo concibe el conocimiento del profesor de forma integrada, pero por su finalidad analítica, lo subdivide en tres dominios: Conocimiento Matemático [MK], Conocimiento Didáctico del Contenido [PCK] y Creencias que permean en cada dominio. Estas últimas no son objeto de estudio en este artículo. Cada dominio posee distintos subdominios, tres en el caso de los dos primeros dominios de conocimiento (Figura 1).

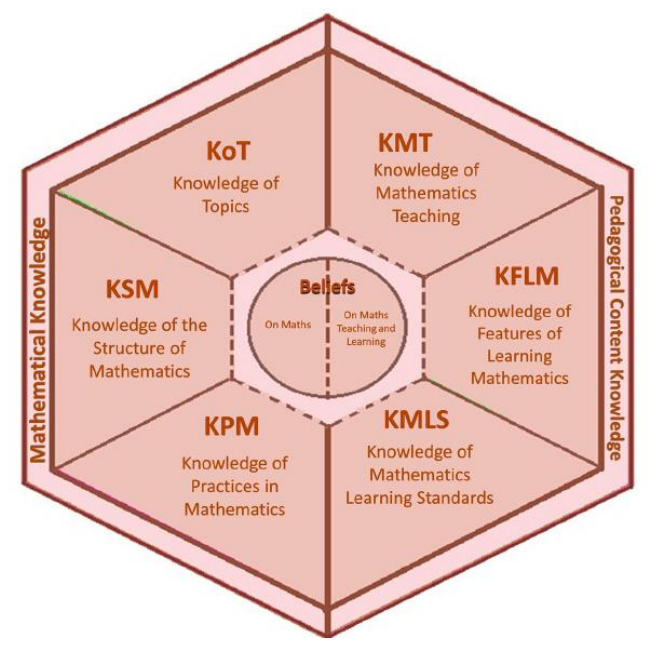

Figura 1 - Subdominios del Mathematics Teacher's Specialised Knowledge [MTSK] Fuente: Carrillo et al., 2018

Por un lado, el dominio del Mathematical Knowledge estudia distintos aspectos del conocimiento matemático del profesor de matemáticas (Carrillo et al., 2018 y su adaptación a la etapa en Muñoz-Catalán, Joglar, Ramírez \& Codes, en prensa, b):

KoT (Conocimiento de los Temas) es más que el conocimiento de la matemática como disciplina. Además de la matemática escolar, consideramos aquí todo conocimiento matemático que le es útil al profesor en su labor. Contiene los procedimientos, la caracterización de conceptos, propiedades de objetos matemáticos y su exhaustividad, distintas formas en las que se puede representar un tema y el conocimiento de usos y aplicaciones de un tema. Serían ejemplos de KoT conocer los atributos relevantes e irrelevantes de los cuerpos geométricos, así como sus definiciones y los registros que se pueden utilizar para representar la descomposición de los cuerpos, así como las especificidades de cada uno de ellos y su complementariedad. 
DOI: 10.20396/zet.v29i00.8661819

KSM (Conocimiento de la Estructura de las Matemáticas) está formado por el conocimiento del profesor sobre las conexiones que permiten una visión general del conocimiento matemático. Este subdominio está formado por tres tipos de conexiones: auxiliares (que permiten hacer un uso instrumental de un contenido en el trabajo con otro contenido), transversales (que subyacen a distintos contenidos matemáticos) y simplificación o complejización, que permiten tanto el conocimiento avanzado desde una perspectiva elemental, como contenido elemental desde una perspectiva avanzada. Mientras que el papel de los dos primeros tipos no parece claro en infantil, las conexiones de simplificación y complejización parece que pueden tener un papel caracterizador del conocimiento especializado de este profesional. Un ejemplo podría ser el conocimiento de que los tres enfoques que tiene el profesor de Educación Infantil acerca de que el trabajar ordenando por tamaños, ayudará al estudio de las escalas.

KPM (Conocimiento de la Práctica Matemática) está formado por el conocimiento del profesor sobre aquellas formas de producir y proceder en matemáticas, incluyendo aspectos de la comunicación matemática, argumentación y prueba que se ponen en juego en la realización de una práctica matemática. Un ejemplo de KPM sería saber que la comparación de cuerpos geométricos es un proceso matemático válido para identificar propiedades de cada cuerpo.

Por otro lado, en el dominio del Pedagogical Content Knowledge se encuentra la caracterización propia de la labor de la enseñanza de las matemáticas. Se consideran los siguientes tres subdominios:

KMT (Conocimiento de la Enseñanza de las Matemáticas) abarca el conocimiento del profesor sobre cómo enseñar un contenido matemático. Incluye conocer distintas estrategias que permitan al profesor impulsar el desarrollo de las capacidades matemáticas procedimentales o conceptuales, las teorías de enseñanza asociadas a un contenido matemático y conocer las características útiles de distintas herramientas para enseñar matemáticas. Sería elemento de KMT conocer la importancia de trabajar desde distintos sistemas de representación (material concreto y fotos desde distintas perspectivas), una determinada composición geométrica (cubo y cilindro), para potenciar el proceso de visualización espacial en los alumnos.

KFLM (Conocimiento de las Características de Aprendizaje de las Matemáticas) que se refiere al conocimiento de cómo se aprende un contenido matemático. Contiene el conocimiento de las dificultades, errores y obstáculos en el aprendizaje de un concepto, así como el conocimiento de la forma en que los alumnos aprenden un cierto contenido. Además de las teorías de aprendizaje asociadas a un contenido matemático y los intereses y expectativas de los estudiantes sobre un contenido matemático. Así, por ejemplo, serían elementos de KFLM el conocimiento de que los alumnos de Educación Infantil requieren situaciones reales y relevantes que dan sentido a la manipulación de objetos o el hecho de que no son capaces de comprender mensajes matemáticos sin apoyo visual. 
DOI: $10.20396 /$ zet.v29i00.8661819

KMLS (Conocimiento de los Estándares de Aprendizaje de las Matemáticas) constituido por todos aquellos referentes que indican en qué momento debe aprenderse cada contenido y a qué nivel de profundidad. Contiene lo que se espera que un estudiante sepa en ese determinado momento escolar, el nivel de desarrollo conceptual o procedimental esperado y la secuenciación con temas anteriores y posteriores. Un ejemplo de KMLS sería conocer qué propiedades de los cuerpos geométricos pueden entenderse en la etapa de Educación Infantil.

\section{Metodología}

En esta investigación nos planteamos comprender el conocimiento especializado que moviliza un profesor de Educación Infantil para enseñar las propiedades de los cuerpos geométricos, identificando elementos de conocimiento especializado y relaciones entre ellos. Esto se lleva a cabo desde la práctica como un lugar privilegiado para comprender cómo este conocimiento sustenta las acciones del profesor. Abordamos dicho objetivo desde un enfoque interpretativo (Bassey, 1999) y utilizando un estudio de caso instrumental (Stake, 2005) que permite comprender en profundidad una realidad singular. Nuestro informante es José (seudónimo), un profesor en activo con más de una década de experiencia en la etapa. José presenta inquietudes por mejorar su práctica, que le llevaron a recibir una formación específica en Didáctica de las Matemáticas.

La recogida de información se ha llevado a cabo a través de observaciones de aula no participantes (videograbaciones) y entrevistas semiestructuradas que se realizan al final de la implementación de la unidad como apoyo para complementar la información aportada por la videograbación. Se efectuaron ocho grabaciones durante dos periodos lectivos consecutivos en un aula de Educación Infantil de un colegio público de la provincia de Sevilla (España). En este estudio se presenta el análisis de la sexta grabación dedicada a geometría, con alumnos de Infantil de 4 años y hacemos referencia a alguna entrevista realizada a José como ayuda a clarificar su práctica desde el conocimiento que sustenta en sus acciones. Con este grupo-clase ha trabajado desde el curso anterior, además del nombre de los cuerpos, algunos elementos como caras, aristas y vértices. Aborda este trabajo mediante distintas estrategias: manipulación directa de los cuerpos, la estampación con pintura y la representación en la pizarra de alguna cara de estos. En el transcurso de las sesiones se establecieron acuerdos de aula sobre el nombre de sus elementos, con la finalidad de ir desligándoles de ese lenguaje cotidiano y orientándolos hacia el vocabulario matemático (p.e. caminitos por aristas y piquitos por vértices). La sesión elegida es inicialmente de repaso de las propiedades de los cuerpos geométricos, aunque se centra concretamente en las caras de estos.

El análisis de los datos se realiza sobre la base de la transcripción de la información, donde aplicamos el enfoque interpretativo (Kvale, 1996). Para este análisis se seleccionan unidades de información en función de si consideramos que constituyen una prueba rotunda del conocimiento del profesor enmarcada en una categoría de un subdominio MTSK (evidencia) o una señal que nos lleva a una determinada categoría pero que hace falta seguir 
DOI: $10.20396 /$ zet.v29i00.8661819

indagando para que sea evidencia (indicio) (Flores-Medrano, 2015). Para analizar utilizamos la caracterización en subdominios y categorías (Carrillo et al., 2018) presentados anteriormente, que nos ayudan a acercarnos a los datos.

Descripción de la sesión de clase objeto de estudio

En este trabajo presentamos la descripción de una sesión de clase en relación con los cuerpos geométricos en la que trabaja sus características e intenta profundizar sobre el número de caras y las figuras geométricas que los conforman. El profesor comienza repasando algunas características: "Vamos a sacar piezas y me tenéis que decir qué son. Vamos a recordar cuántas caras tienen, cuántas aristas tienen. Vamos a recordar si ruedan, cuáles se parecen, cuáles son sus características...”. Como es habitual en sus clases, el profesor utiliza un material manipulativo (piezas de madera) seleccionando los cuerpos de la figura 2, sin dar su nombre.

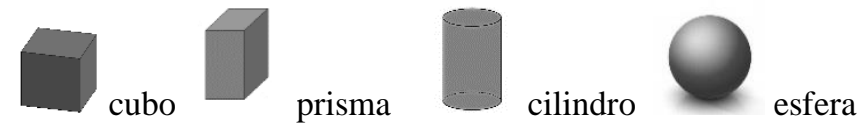

Figura 2. Piezas y nombres específicos de los cuerpos utilizados durante la sesión

Fuente: Elaboración propia

José pregunta en forma de juego “¿En qué mano está? ¿Izquierda o derecha?” y cuando visualizan la pieza, les pregunta "¿Qué es?". Comienza sacando un cubo y, una vez verbalizado el nombre por los alumnos, pasa a escribirlo en la pizarra. A través de preguntas, José centra su atención en las caras de estos: "vamos a poner una pegatina a cada cara para poder contar más fácil”. Lo hace cada vez que dicen un número y al finalizar, les comenta: “¿Cuántas caras hemos dicho que tiene? Vamos a ponerlo aquí (señalando la pizarra). Para saber que pone cara, le vamos a poner una cara" y dibuja una cara en la pizarra y coloca debajo el número seis, comenzando así a realizar una tabla para su registro. A continuación, indica: "En un cubo, las caras ¿Qué son?... ¿Cuántos cuadrados tiene?... ¿Tiene algún rectángulo?". En la tabla, para cada cuerpo, indica número de caras (lo designa con un pictograma) y el número de caras que son cuadrados y rectángulos (designado con cuadrados y rectángulos, respectivamente, en forma estándar) (Figura 3). En el curso de la sesión hay alumnos que se fijan en las aristas, pero José les indica "efectivamente, aristas sí tiene, pero no las vamos a contar. Tiene muchas". El profesor prosigue contando los lados de un cuadrado. A continuación, le ocurre lo mismo con los vértices, donde José les comenta "cada arista termina en un piquito, como nosotros lo llamamos. ¿Cuántos piquitos tiene cada cuadrado?”. 
DOI: $10.20396 /$ zet.v29i00.8661819

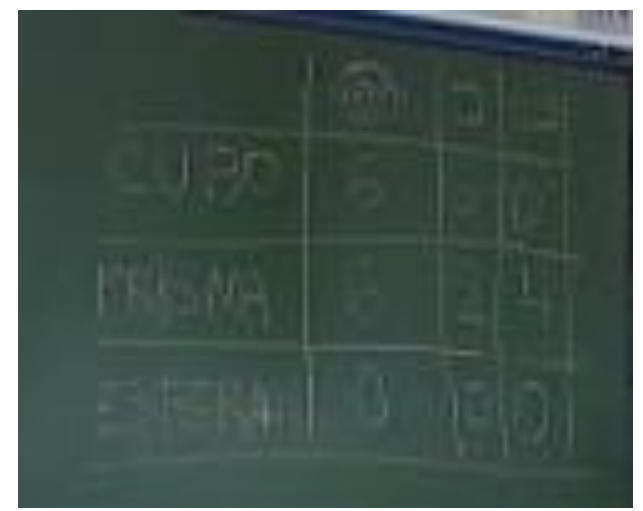

Figura 3 - Representación en la pizarra realizada por el profesor Fuente: Videograbación de clase

Mediante el mismo procedimiento, José se centra en el prisma. Una vez los alumnos visualizan la pieza, la nombran y el profesor escribe el nombre en la pizarra, contabilizan el número de caras que posee, plasmándolo también en la tabla. Tras esto, el profesor destaca: "Seis, igual que el cubo. Pero ahora vamos a ver cuántos cuadrados tiene y cuántos rectángulos. El cubo tenía seis cuadrados, pero no tenía rectángulos. Vamos a ver cuántos cuadrados tiene el prisma. Vamos a ponerle una pegatina a cada cuadrado". Tras el conteo de los cuadrados y su registro en la tabla, José les propone un reto: "Ahora pregunto, a ver quién sabe esto, porque es súper difícil [...] El prisma tiene seis caras, vamos a poner las seis caras, como si fueran niños (mediante pictogramas representa en la pizarra las seis caras, Figura 4) y ¿Cuántas caras son cuadrados?’. Un alumno responde dos y el profesor tacha dos caras de la representación en la pizarra, y les pregunta: “¿Cuántas caras tendrá que son rectángulos?". Ante la respuesta incorrecta de los estudiantes, José vuelve a insistir: "Dos son cuadrados, las demás, hasta llegar a seis, son rectángulos. A ver chicos, ¿Cuántos rectángulos tiene un prisma?". Los estudiantes responden correctamente $\mathrm{y}$, seguidamente, cuenta las caras rectangulares sobre el prisma de madera. Por último, realiza un repaso apoyado en la tabla, dónde, a través de preguntas, compara el cubo y el prisma, haciéndoles ver las similitudes y diferencias entre estos basándose en lo consignado en la tabla.

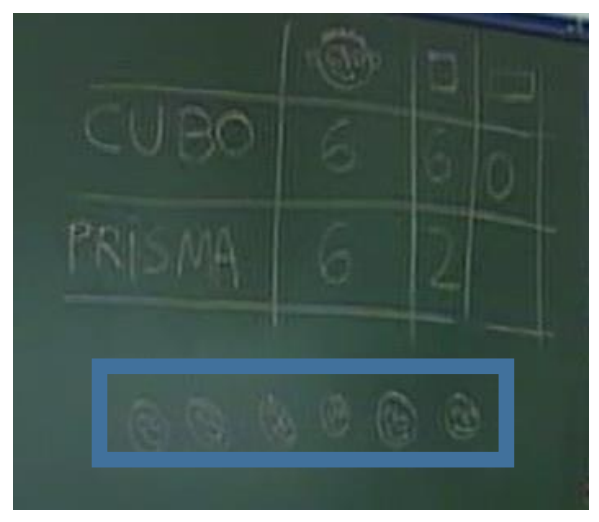

Figura 4 - Representación en la pizarra de las seis caras (como si fueran caras de niños) Fuente: Videograbación de clase 
DOI: $10.20396 /$ zet.v29i00.8661819

A continuación, centra la atención de los estudiantes en la esfera preguntándoles si tiene caras, cuadrados y rectángulos, reflejándolo todo en la tabla. A continuación, aclara "porque si no tiene caras no puede tener ni rectángulos ni cuadrados, ¿Tiene aristas?” y tras la negativa del alumnado, les cuestiona: "Entonces, ¿Qué es lo único que podemos decir de la esfera?", respondiendo estos que rueda.

Para finalizar la sesión, centra su atención en el cilindro, aunque ya esto no lo representa en la pizarra, pues los alumnos se encuentran cansados. Tras la identificación del cuerpo, les pide que indiquen algunas características de estos. Lo primero que destacan es que ruedan, con lo que el profesor interviene preguntándoles "pero ¿por dónde?”. A continuación, uno de los estudiantes indica que tiene círculos y el docente incide en ello: “tiene dos círculos". Inmediatamente, José les pregunta por el número de caras de los cilindros y si estas son cuadrados o rectángulos. Por último, José pregunta sobre las figuras geométricas que componen las caras de estos.

\section{Análisis y Resultados}

En este trabajo presentamos el análisis del conocimiento especializado movilizado por un profesor de Educación Infantil sobre cuerpos geométricos. José pone en práctica lo aprendido en los cursos de mejora realizados, lo que le lleva a trabajar la geometría partiendo de los cuerpos geométricos, en lugar de figuras planas como suele ser habitual. Ya había trabajado con sus alumnos sobre este tema el año anterior, intentando pasar de la identificación del cuerpo a la observación de algunas propiedades: tipo y número de caras, aristas y vértices. En una entrevista nos comenta que su objetivo sobre la enseñanza de la geometría es que los niños discriminen los cuerpos, sean capaces de proporcionar propiedades de estos y no se queden en la mera identificación de su nombre. Comenzó usando la manipulación de los cuerpos y, progresivamente, fue incorporando otros abordajes como la estampación y representación en la pizarra de la cara más representativa para él, por lo que José considera que en este curso de Infantil se debe avanzar hacia la identificación de propiedades (KMLS, expectativas), basándose en su conocimiento de que hay que ayudar a los alumnos a avanzar de su conocimiento de las figuras como un todo (Nivel 1, Van Hiele) a un análisis de las mismas (Nivel 2, Van Hiele) (Jaime y Gutiérrez, 1990) (KFLM, teoría aprendizaje).

En sus clases José trabaja con cuerpos habituales en esta etapa (prisma, cilindro y esfera) ya que considera que son los que deben aprender (KMLS, expectativas). Para él su noción de prima está ligado a tres tipos: cubo, prismas de base cuadrada y rectangular), por tanto, considera que solo el cuadrado y rectángulo pueden formar parte de las caras de estos (KoT, definiciones, propiedades). De esos tres, en clase usa solo los dos primeros: a uno le expresa su nombre particular (cubo) y, al otro, por el nombre genérico de la clase que representa (prisma), lo que se corresponde con el hecho de que acentúe más las diferencias entre las dos figuras que las semejanzas. José valora como algo importante en esta etapa, 
aprender unos cuerpos determinados y sus propiedades (KMLS, nivel de desarrollo esperado).

José conoce que para estos estudiantes es fundamental la manipulación de los cuerpos para comprender sus propiedades (KFLM, formas interacción) y para ello realiza distintas estrategias, orientado a construir una imagen rica de cada cuerpo geométrico y utiliza un material específico de madera (KMT, recursos). Comienza por la manipulación directa, pasando por la estampación y proyección, es decir, utilizando distintos tipos de abordaje (KMT, estrategia) hasta que considera que ya los alumnos pueden trabajar sobre ellos sin necesidad de manipulación directa (KFLM, teoría aprendizaje). Esto ocurre en la sesión analizada (actividad de cierre), donde observamos como es el docente quien manipula los cuerpos y los muestra a los estudiantes, ya que considera que los alumnos han manipulado bastante a través de las distintas estrategias usadas y ya son capaces de responder a las cuestiones con la mera visualización del cuerpo (KFLM, formas interacción). Plantea la actividad para ayudar a los estudiantes a avanzar de Nivel 1 a Nivel 2 de Van Hiele (Jaime et al., 1990), ya que conoce que fijarse, distinguir y reconocer las caras de un cuerpo es algo que les cuesta a los alumnos (KFLM, dificultades), comentándonos en una entrevista que, cuando mostraba un cuerpo a sus alumnos, estos no se fijaban en si tenía cuadrados o rectángulos, sino que se fijaban en el color, en la apariencia, por lo que "las caras son una cosa que los niños no son capaces de distinguir".

El profesor pretende que los estudiantes repasen propiedades como aristas y vértices $\mathrm{y}$, sobre todo, que enriquezcan su conocimiento acerca de las caras de los cuerpos. Para ello José formula una tarea en la que, para cada tipo de cuerpo geométrico, contabilizan el número total de caras y el número de caras en función del tipo de figura plana (cuadrado y rectángulo) (KMT, tarea). José pretende que los estudiantes a esta edad sean capaces de conocer cuántas caras tiene cada cuerpo trabajado (KMLS, expectativas) y la forma que tiene cada cara (KMLS, expectativas). Además, en este procedimiento pretende que los discentes se vayan acostumbrando a los vocablos trabajados (nombres de los cuerpos), tanto mediante un registro de representación oral como escrito, ya que lo considera contenido a trabajar para esta edad (KMLS, expectativas).

El tipo de tarea que implementa refleja su conocimiento acerca del uso de diferentes formas de representación como vehículo para el desarrollo de la sesión, usándolo como estrategia de enseñanza (KMT, estrategia). José muestra su conocimiento de distintos registros de representación en el ámbito de la geometría (manipulativo [piezas de madera], oral, escrito, simbólico [cuando escribe el número 6] y gráfico [tabla]) (Lesh, Post \& Behr, 1987) y la complementariedad entre ellos (KoT, registro representación). Además, valora la tabla como un registro útil para recoger información de manera ordenada y promover una posterior interpretación (KoT, registro representación).

En la tabla utiliza distintos tipos de designación (pictograma y símbolo "6") sabedor de la importancia que tiene la designación en la génesis de contenidos matemáticos (KoT, propiedades). Estos aspectos suponen un indicio de que el profesor conoce que el uso de 
pictogramas y lenguaje cotidiano le acerca al concepto matemático, ayudándoles en su aprendizaje matemático (KMT, estrategia). Cuando se le pregunta a José en las entrevistas por definiciones de cuerpos concretos suele proporcionarlas sobre la base de un listado de elementos constituyentes, que distan de las características de una definición formal de las mismas. Este modo de definir se corresponde con cómo concibe que se inicia el trabajo sobre la definición en Infantil, que es ayudando a los alumnos a identificar sus propiedades (KPM, definición), sabedor del modo en que razonan geométricamente los niños en esta etapa (KFLM, teoría aprendizaje).

Una vez registrada la información en la tabla, el profesor promueve mediante preguntas la comparación de las propiedades de los cuerpos (KMT, estrategia). La comparación se convierte en una práctica a través de la cual, mediante las diferencias y similitudes entre los cuerpos, los alumnos profundizan en el conocimiento de estos, identificando sus propiedades (KPM, forma de génesis epistemológica). El profesor conoce que es más fácil para ellos partir de un objeto que ellos ya conocen, sacando similitudes y diferencias con el nuevo objeto (KFLM, formas interacción). José conoce la importancia de mostrar el tipo de caras de los cuerpos pues considera que ayuda a los estudiantes a diferenciar el cubo del prisma cuadrangular regular (KMT, estrategia). En sus clases, José comienza utilizando vocabulario propio del niño para referirse a algunas propiedades de los cuerpos como, por ejemplo, llama "caminito" a la arista, "piquito" al vértice y comenta "que rueda" cuando se refiere a que posee una cara curva (KMT, lenguaje como herramienta de enseñanza). En el curso de la sesión se observa como José valora la adquisición de vocabulario geométrico específico y el uso de lenguaje correcto y preciso (KMLS, expectativas).

Como hemos dicho, en la sesión José plantea el estudio de los cuerpos geométricos mediante el conteo de sus caras. El profesor pretende trabajar las caras de distintos cuerpos (cubo, prisma, esfera y cilindro) mediante un mismo procedimiento de enseñanza, comenzando por mostrar la pieza, colocar el nombre en la pizarra, contar el total de caras y contar el número de caras que son cuadrados y rectángulos (KMT, técnica). El profesor conoce las necesidades de sus estudiantes y se va adaptando a ellas, por lo que va tomando decisiones en sus clases en función del conocimiento que posee sobre su alumnado (KMT, estrategia). Por ejemplo, para el trabajo del cilindro opta por no representar en la pizarra pues encuentra al alumnado ya bastante cansado. Para el recuento de caras va colocando una pegatina cada vez que dicen un número, lo que muestra una conversión entre dos registros de representación de un mismo concepto (pictórico-simbólico de la cantidad) (KoT, registros de representación). Además, refleja su conocimiento sobre la enumeración como procedimiento para contar (KoT, procedimientos). Y, por otro lado, conoce que el pegar pegatinas sirve como estrategia para contar el número de caras iguales en un cuerpo geométrico, asociando una pegatina a cada cara para facilitar el recuento (KMT, recursos).

En el recuento de las caras del prisma, usa pegatinas para contabilizar la totalidad de caras y las caras cuadradas, pero para el recuento de caras rectangulares deja de lado el material manipulable, animándolos a la abstracción. Plantea otra forma de contar el número de caras, 
presentando de manera verbal un problema aditivo parte-todo con la incógnita en una de las partes (KoT, procedimientos). El profesor conoce de la importancia de la resolución de problemas en la actividad matemática escolar (KMLS, expectativa) y que los estudiantes tienen dificultades a la hora de manejar cantidades numéricas sin el apoyo de material manipulativo (KFLM, dificultades), por lo que termina cambiando a un registro pictóricográfico, donde representa en la pizarra las seis caras y tacha dos de ellas (KoT, registros de representación). Con este planteamiento también podemos decir que conoce de la importancia de utilizar distintos contextos (KoT, fenomenología). Seguidamente, el profesor plantea la comprobación del resultado mediante el conteo físico de las caras rectangulares en la pieza de madera (KPM, comprobación), valorándola como como fase relevante en la resolución de problemas (KPM, resolución de problemas).

Aunque el docente pretende trabajar en esta sesión solo las caras, los alumnos están acostumbrados a tratar también aristas y vértices cuando trabajan los cuerpos, por lo que ellos intentan centrarse en estos. En el caso de las aristas, el profesor les dice "no las vamos a contar. Tiene muchas", posiblemente porque considera que los números que se manejan a estas edades no sobrepasan la decena (KMLS, nivel de desarrollo esperado). Por otro lado, podemos decir que sea debido a que no pretende dedicar esta sesión a profundizar sobre ello, sino que lo van a trabajar posteriormente, pudiendo ser solo una decisión de enseñanza (KMLS, secuenciación). Acto seguido les pregunta por la cantidad de lados que tiene un cuadrado, haciendo de nuevo referencia a las caras, lo que nos hace pensar que lo realiza, no porque tiene muchas sino porque no le interesa centrarse en esas propiedades. Esta forma de redirigir la clase hacia lo que le interesa, nos muestra otra estrategia de enseñanza (KMT, estrategia). Con la expresión "cada arista termina en un piquito" observamos como la manera de enfocar la arista es dinámica porque hace referencia a la magnitud longitud (KoT, propiedades) y esto a su vez lo lleva a la enseñanza (KMT, estrategia). Y, por último, también podemos decir que tenemos indicios de conocimiento acerca de la consideración de la contabilización de lados y vértices del cuadrado como contenido para la edad (KMLS, expectativas).

\section{Conclusiones}

La investigación sobre matemáticas en la Etapa de Educación Infantil es un tema en auge (Charalambous \& Pitta-Pantazi, 2016). McCray, et al. (2012) revelan que los conocimientos adquiridos en esa etapa constituyen la base para aprendizajes matemáticos futuros, lo que pone de manifiesto la relevancia de una buena formación matemática en este nivel. Las matemáticas de la etapa contienen ideas profundas (Perry et al., 2002) por lo que el profesor necesita poseer un conocimiento matemático sólido y cohesionado (Muñoz-Catalán, et al., 2017).

Aunque este profesional es diferente a los profesores de Educación Primaria, Secundaria, Bachillerato o Universidad, la distinción del modelo MTSK en los tres grandes dominios e incluso en cada uno de sus respectivos subdominios puede aplicarse en él. Este 
trabajo nos permite extraer conclusiones que respaldan y concretan el conocimiento especializado de un profesor de Educación Infantil en la enseñanza de cuerpos geométricos. El uso de distintas herramientas metodológicas simultáneas como categorías, subdominios de MTSK y los constructos evidencia e indicio nos ha servido para obtener una mejor comprensión sobre el conocimiento especializado de este docente.

Como señalábamos en Muñoz-Catalán, et al. (2019) el conocimiento del profesor para enseñar geometría es diferente al requerido para enseñar números. El análisis refleja evidencias e indicios de casi todos los subdominios de conocimiento, excepto de KSM, ya que es un subdominio difícil de encontrar en una observación de aula (Escudero-Domínguez, et al., 2019). En el análisis hemos observado como su finalidad es avanzar hacia la identificación de propiedades de los cuerpos geométricos, destacando su conocimiento sobre las nociones que considera que los alumnos deben aprender en este curso (KMLS), así como su conocimiento acerca del cómo ayudar al alumnado en el avance de su aprendizaje matemático, acercándole los conceptos (KFLM) y tomando diversas decisiones de enseñanza (KMT). Por otro lado, hemos observado el importante papel que toman los distintos registros de representación en la sesión (KoT) y como estos se muestran decisivos en su enseñanza (KMT) para favorecer la comprensión de un concepto. En esta sesión usa la tabla como registro que permite organizar la información y poder realizar después su interpretación. El lenguaje es otro sistema de representación importante en la enseñanza de las matemáticas de Infantil (KoT), ya que es una etapa generadora de lenguaje. José comienza utilizando términos del lenguaje cotidiano que transmiten la idea asociada al concepto (KMT), pero una vez que ya han captado el significado de la noción, va introduciendo progresivamente el lenguaje matemático, por lo que valora la adquisición de vocabulario geométrico específico y el uso de lenguaje correcto y preciso (KMLS).

A raíz del análisis de este caso seguimos teniendo la necesidad de poner en valor el subdominio de KPM. Como se indica en Muñoz-Catalán et al. (en prensa, b) para conocer cómo se razona y procede para llegar a resultados matemáticos y cómo se valida es necesario para un buen desempeño profesional. Sin embargo, nos exige adoptar una nueva mirada sobre las prácticas matemáticas en el aula de Infantil que permitan concebirlas como formas de génesis epistemológica (Muñoz-Catalán et al., en prensa, a). Así, podríamos destacar la comparación como una manera de generar conocimientos geométricos a esta edad y que es usada como estrategia de enseñanza (KMT). Consideramos que un buen conocimiento en este subdominio le proporcionaría más profundidad en el diseño de tareas y la gestión de la actividad matemática que se produce en el aula.

Este trabajo reafirma la necesidad de un sólido conocimiento especializado en el dominio matemático, así como la relación de estos con elementos de conocimiento didáctico del contenido, es decir, sabemos que lo que realiza en sus clases es sustentado por su conocimiento matemático (Escudero-Domínguez, et al., en prensa). Entre la diversidad de relaciones entre distintos subdominios de conocimiento existentes en este análisis resaltamos la relación KPM - KMT. Por ejemplo, el conocimiento de la comparación como práctica matemática que es usada como estrategia de enseñanza; el conocimiento sobre el uso de 
DOI: $10.20396 /$ zet.v29i00.8661819

lenguaje correcto y preciso en el aprendizaje geométrico que es usado como herramienta de enseñanza. Estas relaciones respaldan la naturaleza compleja e integral del conocimiento especializado. El tipo de tarea propuesta, el material manipulable, la interacción en el aula, el realizar recuentos de elementos de los cuerpos y el establecimiento de relaciones entre ellos son promotoras de procesos de razonamiento espacial (Gamboa, et al., 2010). También hemos sentido dificultad a la hora de distinguir si los elementos de conocimiento especializado identificados eran evidencias o indicios, especialmente en el dominio matemático (Muñoz-Catalán, Joglar-Prieto, Ramírez-García \& Liñán-García, 2019).

Las investigaciones que abordan el conocimiento del profesor de Educación Infantil son escasas y concluyen que las características propias de la etapa deben tenerse en cuenta (Mosvold, et al., 2011). Además, este trabajo, junto con otros como Muñoz-Catalán et al. (2019), pone en valor la importancia que tiene el conocimiento de carácter pedagógico general del docente en la etapa, por el desarrollo cognitivo y físico de los discentes. Por ejemplo, José usa pictogramas para acercarles el concepto; va, poco a poco, utilizando más información matemática; se adapta a las necesidades de sus estudiantes (cansancio, comprensión matemática, ...) en cada una de sus clases. Como prospectiva pensamos que sería interesante ahondar sobre la conjunción del conocimiento pedagógico general con el conocimiento especializado para albergar más información que nos ayude a comprender mejor el conocimiento que este profesional necesita para ejercer su profesión.

Las aportaciones de este trabajo se centran también en el fortalecimiento de la preparación matemática de estos profesionales, con el objetivo de armar la consistencia del conocimiento que necesitan para gestionar el aprendizaje de los estudiantes. El gran peso que adquiere la parte del conocimiento matemático contrasta con la perspectiva que se tiene del profesor de Educación Infantil pues a lo largo de su carrera profesional no se les suele exigir una formación profunda y específica en matemáticas (Muñoz-Catalán et al., 2019). Algunas asociaciones (p.e. NAEYC \& NCTM, 2013) han señalado la necesidad de proporcionar una formación matemática sólida en esta etapa, con lo que los docentes deben entender qué tipo de problemas matemáticos tienen que resolver y qué tipo de recursos matemáticos manejar para resolver esos problemas (Bass, 2005).

\section{Agradecimientos}

Este trabajo desarrollado en el marco del proyecto: "Conocimiento especializado del profesorado de matemáticas y formación del profesorado" (RTI2018-096547-B-I00, del Ministerio de Ciencia, Innovación y Universidades del Gobierno de España). Asimismo, está vinculado a la Red MTSK de la Asociación Universitaria Iberoamericana de Posgrado (AUIP) Este trabajo desarrollado en el marco del proyecto: "Conocimiento especializado del profesorado de matemáticas y formación del profesorado" (RTI2018096547-B-I00, del Ministerio de Ciencia, Innovación y Universidades del Gobierno de España). Asimismo, está vinculado a la Red MTSK de la Asociación Universitaria Iberoamericana de Posgrado (AUIP). 


\section{Referencias}

Ball, D.L., Thames, M.H., \& Phelps, G. (2008). Content Knowledge for Teaching. What Makes It Special? Journal of Teacher Education, 59 (5), 389-407.

Bass, H. (2005). Mathematics, mathematicians, and mathematics education. Bulletin of the American Mathematical Society, 42, 417-430.

Bassey, M. (1999). Case study research in educational settings. Buckingham: Open university press.

Carrillo, J., Climent, N., Montes, M., Contreras, L.C., Flores-Medrano, E., Escudero-Ávila, D., ... Muñoz-Catalán, M.C. (2018). The Mathematics Teacher's Specialised Knowledge (MTSK) model. Research in Mathematics Education, 20 (3), 236-253.

Castro, E. \& Castro, E. (Coords.) (2016). Enseñanza y aprendizaje de las matemáticas en educación infantil. Madrid: Pirámide.

Charalambous, C., \& Pitta-Pantazi, D. (2016). Perspectives on Priority Mathematics Education: Unpacking and Understanding a Complex Relationship Linking Teacher Knowledge, Teaching, and Learning. En L.D. English y D. Kirshner (Eds.), Handbook of International Research in Mathematics Education (pp. 19-59). New York, NY: Routledge.

Clements, D. H., \& Sarama, J. (2011). Early childhood teacher education: the case of geometry. Journal of Mathematics Teacher Education, 14, 133-148. https://doi.org/10.1007/s10857-011-9173-0

Escudero-Domínguez, A., Muñoz-Catalán, M.C., \& Carrillo, J. (en prensa). Caracterizando el Conocimiento Especializado de un Profesor de Educación Infantil Enseñando Prismas. En (Eds.), Investigación en Educación Matemática XXIV. Valencia: SEIEM.

Escudero-Domínguez, A., Escudero-Ávila, D., Aguilar-González, A., \& Vasco-Mora, D. (2019). El conocimiento especializado del profesor de matemáticas en Educación Infantil para la enseñanza de Geometría. En J. Carrillo, M. Codes y L.C. Contreras (Eds.), IV Congreso Iberoamericano sobre Conocimiento Especializado del Profesor de Matemáticas (pp. 219-227). Huelva: Universidad de Huelva Publicaciones.

Escudero-Domínguez, A., Muñoz-Catalán, M.C., \& Carrillo, J. (2017). MTSK: Conocimiento especializado para la enseñanza de la geometría en la etapa infantil. En J. Carrillo y L.C. Contreras (Eds.), Avances, utilidades y retos del modelo MTSK. Actas de las III Jornadas del Seminario de Investigación de Didáctica de la Matemática de la Universidad de Huelva (pp. 119-124). Huelva: CGSE.Flores-Medrano, E. (2015). Una profundización en la conceptualización de elementos del modelo de Conocimiento Especializado del Profesor de Matemáticas (MTSK). Tesis doctoral. Huelva, España: Universidad de Huelva.

Gamboa, R., \& Ballestero, E. (2010). La enseñanza y aprendizaje de la geometría en secundaria, la perspectiva de los estudiantes. Revista Electrónica Educare, vol. XIV, 2, 125-142. Universidad Nacional Heredia, Costa Rica.

Hundeland, P.S., Erfjord, I., \& Carlsen, M (2017). A kindergarten teacher's revealed knowledge in orchestration of mathematical activities. In Proceedings of the Tenth 
DOI: $10.20396 /$ zet.v29i00.8661819

Congress of the European Society for Research in Mathematics Education (CERME 10). TWG13: Early years mathematics, (pp. 1853 - 1860). Feb 2017, Dublin, Ireland.

Jaime, A., \& Gutiérrez, A. (1990). Una propuesta de fundamentación para la enseñanza de la Geometría: El modelo de van Hiele. En S. Llinares y M. Sánchez (Eds.), Teoría y práctica en educación matemática (pp. 295-384). Sevilla: Alfar.

Kvale, S. (1996). Interviews: An introduction to qualitative research interviewing. Londres: SAGE.

Lesh, R., Post, T., \& Behr, M. (1987). Representations and translations among representations in mathematics learning and problem solving. En C. Janiver (Ed.), Problems of representation in the teaching and learning of mathematics (pp. 33-40). Hillsdale, NJ: Lawrence Erlbaum.

McCray, J.S., \& Chen, J-Q. (2012). Pedagogical Content knowledge for preschool mathematics: construct validity of a new teacher interview. Journal of Research in Childhood Education, 26, 291-307.

Mosvold, R., Bjuland, R., Fauskanger, J., \& Jakobsen A. (2011). Similar but different investigating the use of MKT in a Norwegian kindergarten setting. En M. Pytlak, T. Rowland, y E. Swoboda (Eds.), Proceedings of the Seventh Congress of the European Society for Research in Mathematics Education (pp. 1802-1811). Rzeszów: University of Rzeszów.

Muñoz-Catalán, M.C., Joglar, N., Ramírez, M., \& Codes, M. (en prensa, b).

Muñoz-Catalán, M.C., Joglar-Prieto, N., Ramírez-García, M., \& Liñán-García, M.M. (2019). MTSK en Educación Infantil. En J. Carrillo, M. Codes y L.C. Contreras (Eds.), IV Congreso Iberoamericano sobre Conocimiento Especializado del Profesor de Matemáticas. Huelva: Universidad de Huelva Publicaciones.

Muñoz-Catalán, C., Joglar, N., Ramírez, M., Escudero, A.M., Aguilar, A., \& Ribeiro, M. (2019). El conocimiento especializado del profesor de infantil desde el aula de matemáticas. En E. Badillo, N. Climent, C. Fernández y M. T. González (Eds.), Investigación sobre el profesor de matemáticas: formación, práctica de aula, conocimiento y competencia profesional (pp. 63-84). Salamanca: Ediciones Universidad Salamanca.

Muñoz-Catalán, M.C., Liñán-García, M.M., \& Ribeiro, M. (2017). El conocimiento especializado para enseñar la operación de resta en Educación Infantil. Cadernos de Pesquisa, 24, 4-19.

Muñoz-Catalán, M.C., Ramírez-García, M., Joglar-Prieto N., \& Carrillo J. (en prensa, a). Mathematics Teacher's Specialised Knowledge to promote Algebraic Reasoning in Early Childhood Education as from a task of additive decomposition. Infancia y Aprendizaje Journal for the Study of Education and Development.

NAEYC, \& NCTM (2013). Matemáticas en la Educación Infantil: Facilitando un buen inicio. Declaración conjunta de posición. Edma 0-6: Educación Matemática en la Infancia, 2(1), 1-23.

Parks, A.N. \& Wager, A.A. (2015). What Knowledge is Shaping Teacher Preparation in Early Childhood Mathematics? Journal of Early Childhood Teacher Education, 36 (2), 124-141. 
DOI: $10.20396 /$ zet.v29i00.8661819

Perry, B., \& Dockett, S. (2002). Young children's access to powerful mathematical ideas. En L.D. English (Ed.), Handbook of international research in mathematics education (pp. 81-112). Mahwah, NJ: Lawrence Erlbaum Associates.

Pitta-Pantazi, D., \& Christou, C. (2011). The structure of prospective kindergarten teachers' proportional reasoning. Journal of Mathematics Teacher Education, 14, 149-169.

Policastro, M., Almeida, A., \& Ribeiro, M. (2017). Conhecimento especializado revelado por professores da Educação Infantil e dos Anos Iniciais no tema de Medida e Comprimento e sua Estimativa. Revista Plural, 17(36), 123-154

Rowland, T., Huckstep, P., \& Thwaites, A. (2005). Elementary Teachers' Mathematics Subject Knowledge: The Knowledge Quartet and the Case of Naomi. Journal of Mathematics Teacher Education, 8, 255-281.

Scheiner, T., Montes, M.A., Godino, J.D., Carrillo, J., \& Pino-Fan, L.R. (2019). What Makes Mathematics Teacher Knowledge Specialized? Offering Alternative Views. International Journal of Science and Mathematics Education, 17(1), 153-172.

Shulman, L. (1986). Those who understand: Knowledge growth in teaching. Educational Researcher, 15 (2), 4-14.

Stake, R.E. (2005). Multiple Case Study Analysis. New York: Guilford Press.

Wernberg, A., Larsson, K., \& Riesbeck, E. (2010). Matematik iförskolan (Mathematics in preschool). En B. Riddersporre, y S. Persson (Eds.), Utbildningsvetenskap för förskolan (Educational sciences for preschool) (pp. 157-171). Stockholm, Sweden: Natur y Kultur.

Zakaryan, D., \& Ribeiro, M. (2016). Conocimiento de la enseñanza de números racionales: una ejemplificación de relaciones. Zetetiké, 24(3), 301-321. 